\title{
Nonlinear Switching in an All-Semiconductor-Optical-Amplifier Loop Device
}

\author{
Jiun-Haw Lee, Ding-An Wang, Hsin-Jiun Chiang, Ding-Wei Huang, Steffen Gurtler, \\ C. C. Yang, Yean-Woei Kiang, B. C. Chen, M. C. Shih, and T. J. Chuang
}

\begin{abstract}
An all-semiconductor-optical-amplifier loop device with a multimode interference (MMI) coupler was fabricated with the deep UV cryo-etching technique. Efficient power-dependent switching was observed. With continuous-wave signals, nonlinear switching occurred due to the combined effect of the nonlinear coupling in the MMI coupler and the lateral wave field redistribution caused by the loop structure. Simulation results showed good agreement in trend with the experimental data.
\end{abstract}

Index Terms-All-optical switching, nonlinear optical loop mirror, semiconductor optical amplifier.

\section{INTRODUCTION}

$\mathbf{N}$ ONLINEAR optical loop mirrors (NOLM's) have received much attention for fast all-optical switching with the applications to high-speed signal processing and optical communications. Typically, such a device consists of either an all-fiber loop or a fiber loop asymmetrically inserted with a semiconductor optical amplifier [1]-[5]. The fiber loop is connected with a fiber coupler for input/output coupling. The required optical nonlinearity for all-optical switching comes from either the Kerr effect in fiber or gain saturation in the amplifier. The fiber loop length of such a device is at least a couple meters which corresponds to a latency of around $10 \mathrm{~ns}$ ! Recently, an all semiconductor NOLM with one small section of the loop being active was fabricated to demonstrate $20-\mathrm{Gb} / \mathrm{s}$ demultiplexing [6]. With the miniature structure, the latency of the device can be tremendously reduced.

In this letter, we report the experimental and simulation results of nonlinear switching in an all-semiconductor-opticalamplifier loop device. For input/output coupling, a multimode interference (MMI) amplifier was connected to the loop. It was found that the observed nonlinear switching comes from the combined effect of the nonlinear coupling in the MMI amplifier and the lateral wave field redistribution caused by the

Manuscript received September 28, 1998; revised October 21, 1998. This work was supported by the National Science Council, R.O.C., under Grant NSC 87-2215-M-002-025, Grant NSC 87-2215-E-002-006, and Grant NSC 87-2215-E-002-007.

J.-H. Lee, D.-A. Wang, H.-J. Chiang, D.-W. Huang, S. Gurtler, C. C. Yang, and Y.-W. Kiang are with the Department of Electrical Engineering and Graduate Institute of Electro-Optical Engineering, National Taiwan University, Taipei, Taiwan, R.O.C.

B. C. Chen is with the Department of Chemistry, National Taiwan University, Taipei, Taiwan, R.O.C.

M. C. Shih is with the Institute of Opto-Electrical Sciences, National Taiwan Ocean University, Keelung, Taiwan, R.O.C.

T. J. Chuang is with the Institute of Atomic and Molecular Sciences, Academia Sinica, Taipei, Taiwan, R.O.C.

Publisher Item Identifier S 1041-1135(99)01111-8.

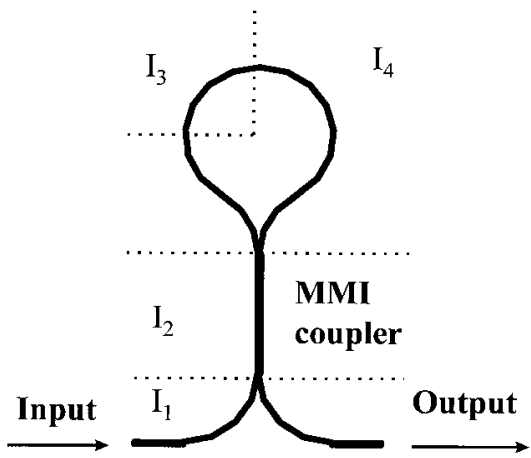

Fig. 1. Schematic diagram of the all-semiconductor-optical-amplifier loop device. Electrodes are divided into four sections denoted by $I_{1}-I_{4}$.

loop. This mechanism is different from that in an NOLM in which the nonlinear optical mechanism comes from the loop. This device has a loop length of only $2.888 \mathrm{~mm}$ (including the coupler), corresponding to a latency of only $31.8 \mathrm{ps}$. Also, such an all semiconductor device can be easily integrated monolithically with other optical devices and exhibits the advantages of compactness, low cost and mass production. Meanwhile, with the all-active structure it is expected that the switching intensity can be reduced and the fanout can be increased.

Fig. 1 shows the layout of the device. The loop of $300 \mu \mathrm{m}$ in radius is formed with a curved ridge-loading waveguide with a ridge width $4 \mu \mathrm{m}$ (expected to form a single-mode waveguide). The loop is connected to an MMI waveguide with a length $520 \mu \mathrm{m}$ and a ridgewidth $8 \mu \mathrm{m}$. This MMI waveguide serves the function of the coupler. Then, the input and output legs are formed with $4-\mu \mathrm{m}$-wide waveguides. Both have the lengths of about $100 \mu \mathrm{m}$. The semiconductor optical amplifiers were fabricated on a four-period GaAs-AlGaAs multiple-quantum-well (MQW) epitaxial structure. The GaAs wells $\left(10 \mathrm{~nm}\right.$ thick) and $\mathrm{Al}_{0.25} \mathrm{Ga}_{0.75}$ As barriers $(20 \mathrm{~nm})$ were sandwiched by two 140 -nm-thick i- $\mathrm{Al}_{0.25} \mathrm{Ga}_{0.75}$ As separate confinement layers, and p- $\mathrm{Al}_{0.6} \mathrm{Ga}_{0.4} \mathrm{As}$ and $\mathrm{n}-\mathrm{Al}_{0.6} \mathrm{Ga}_{0.4} \mathrm{As}$ cladding layers. The fabrication procedures were the same as the typical processes for semiconductor lasers except the etching technique for forming the ridge-loading waveguides. To achieve vertical and smooth etched walls for reducing bending loss and to minimize surface damage, we used the UV-assisted cryo-etching technique [7]. The etching depth was $1.8 \mu \mathrm{m}$. For injecting different currents into different areas, we divided the electro-pad into four disconnected regions, 


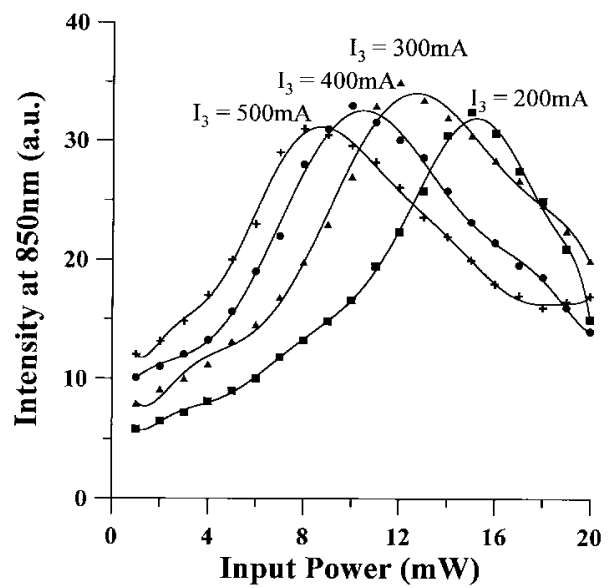

Fig. 2. Output power level as a function of input power with different $I_{3}$ values: $I_{1}=100 \mathrm{~mA}, I_{2}=60 \mathrm{~mA}$, and $I_{4}=250 \mathrm{~mA}$.

indicated by the injection currents $I_{1}$ (input-output region), $I_{2}$ (MMI coupler), $I_{3}$ (one-quarter of the loop) and $I_{4}$ (threequarters of the loop).

Fig. 2 demonstrates four sets of data showing powerdependent switching. We used a continuous-wave (CW) Ti:sapphire laser at $850 \mathrm{~nm}$ with various power levels as input signals and monitored the output power variation at the same wavelength. The horizontal axis shows the input power before entering the input waveguide. Because the guiding layer of the amplifier waveguide was quite thin (only about $0.3 \mu \mathrm{m}$ ), the input coupling efficiency was estimated to be only on the order of $0.1 \%$ or even smaller. In this measurement, $I_{1}=100 \mathrm{~mA}, I_{2}=60 \mathrm{~mA}, I_{4}=250 \mathrm{~mA}$ and $I_{3}$ was varied from 200 to $500 \mathrm{~mA}$ as shown in the figure. These curves show the depression of output power level after the input power reaches a certain value. This behavior is similar to the power-dependent switching observed in an NOLM. Note that the maximum output is obtained at a smaller input power level with a larger $I_{3}$ value. Within the data window, we have achieved an output contrast ratio as large as 2.36 between the input levels 10 and $20 \mathrm{~mW}$ when $I_{3}$ was $400 \mathrm{~mA}$. Fig. 3 demonstrates three sets of data with $I_{2}$ varied from 40 to 80 $\mathrm{mA}$. In this figure, $I_{1}, I_{3}$, and $I_{4}$ were fixed at 100, 300 and $250 \mathrm{~mA}$, respectively. The $I_{2}$ value was somewhat critical for achieving the nonlinear behavior. The power dependent switching effect was quite significant when $I_{2}$ was $60 \mathrm{~mA}$. When $I_{2}$ was lower than $30 \mathrm{~mA}$ or higher than $90 \mathrm{~mA}$, no significant nonlinear effects were observed. Also, we can see that with a larger $I_{2}$, the nonlinear switching occurs at a lower input power level.

To understand the mechanisms behind the power-dependent switching, we conducted simulations on the device operation. Since we used CW signals in our experiments, the functions of the loop were to amplify the counter-propagating signals and to rearrange the lateral wave field distribution in the MMI waveguide. It did not contribute to the nonlinear optical mechanism for the function of an NOLM. With CW signals the nonlinear optical mechanism for the observed powerdependent switching comes from the MMI coupler. For the MMI coupler, we solved a set of equations for the forward- and

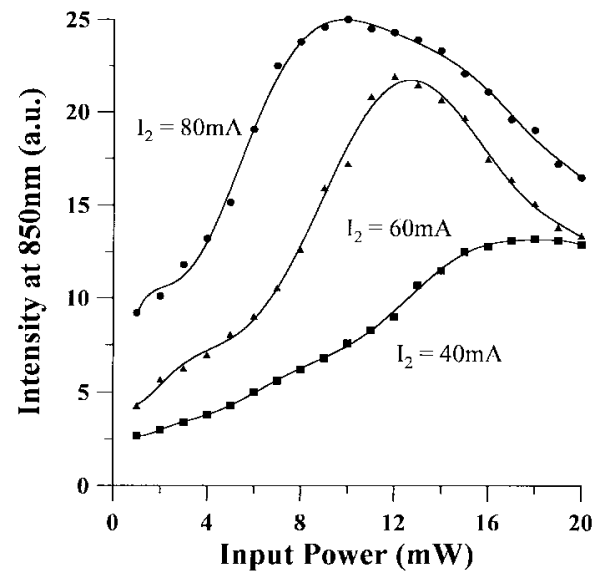

Fig. 3. Output power level as a function of input power with different $I_{2}$ values: $I_{1}=100 \mathrm{~mA}, I_{3}=300 \mathrm{~mA}$, and $I_{4}=250 \mathrm{~mA}$.

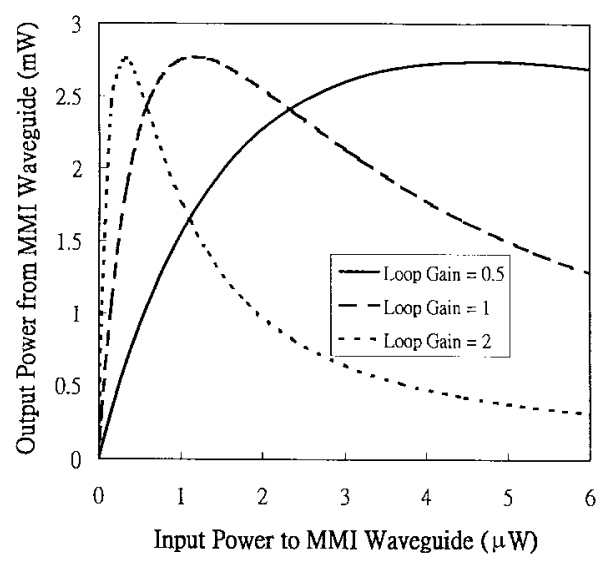

Fig. 4. Simulation results with the variation of the loop gain factor from 0.5 to 2 .

backward-propagating wave fields which consumed the same carrier density distribution. With typical parameter values in a GaAs-AlGaAs optical amplifier and those of the experimental conditions, the simulation results shown in Figs. 4 and 5 were obtained. In the simulations, the amplifiers of the input and output legs were ignored. In both Figs. 4 and 5, the powerdependent switching results, just like those in the experimental results, can be seen. In Fig. 4, the injection current into the MMI coupler is fixed at $100 \mathrm{~mA}$ and the three curves represent the cases of gain factor from the loop as 2 (dotted), 1 (dashed) and 0.5 (solid), respectively. The loop gain factor depends on the bending loss of the waveguide and the injection currents $I_{3}$ and $I_{4}$. Therefore, it can be represented by the level of $I_{3}$. Hence, Fig. 4 can be compared with Fig. 2 qualitatively. Although a good explanation for the details of the simulation results requires further numerical study, the simulation results agree well in trend with the experimental data. In particular, the right-shift trends of the output maximum with decreasing loop gain factor and $I_{3}$ coincide very well. Similar comparison can be made between Figs. 3 and 5, in which the injection current $I_{2}$ into the MMI coupler is varied. Although the variation ranges of the injection currents are quite different, the two figures show the same trend, i.e., the output maximum right-shifts with decreasing $I_{2}$. 


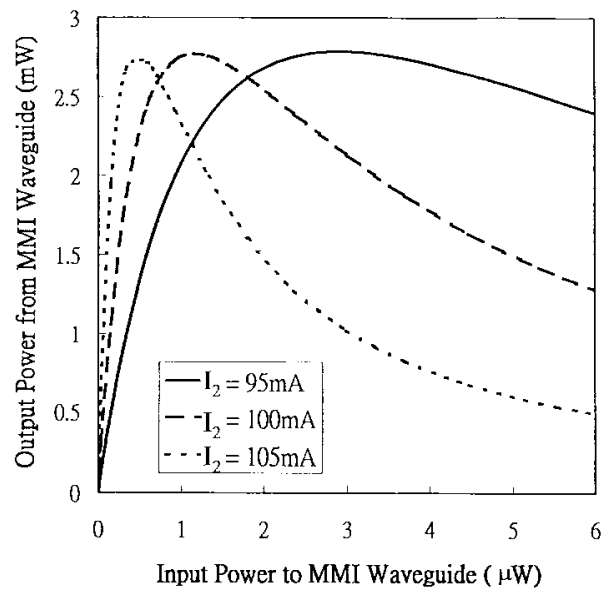

Fig. 5. Simulation results with the variation of the injection current into the MMI coupler from 95 to $105 \mathrm{~mA}$.

An MMI waveguide has been used for nonlinear switching with output emerging from either one-half side or the other of the lateral dimension of the waveguide, depending on the input power level [8]. Typically, when the MMI waveguide has a length close to one-half the beat length, a low-power signal entering the waveguide from one one-half side would emerge from the other one-half side. However, the major part of a high-power input signal would emerge from the same onehalf side. Such a phenomenon was usually called the nonlinear coupling in a zero-gap directional coupler. With an MMI waveguide amplifier, the mechanisms leading to nonlinear coupling are gain saturation and the induced refractive-index change when the signal duration is longer than several ps. In our situation, after the $\mathrm{CW}$ input signal enters the MMI coupler from the side of the input leg, the lateral distribution of signal field at the end of the loop depends on the input power level and the injection current $I_{2}$. With a higher injection current and hence higher gain, gain can be more easily saturated and hence the nonlinear effects can be more easily observed. The loop plays the role of exchanging the wave field distribution between the two one-half sides of the MMI coupler besides providing gain (controlled by $I_{3}$ and $\left.I_{4}\right)$. It was numerically found that the exchange of wave field distribution was important for efficient nonlinear switching. After the signals return to the MMI coupler from the loop, the evolution of the lateral field distribution again depends on the power level and the injection current $I_{2}$. Therefore, the power level from the output leg shows the nonlinear dependence on the input power. This dependence varies with the injection currents.

In conclusion, we have fabricated an all-semiconductoroptical-amplifier loop device and characterized its operation with $\mathrm{CW}$ signals. With the loop to redistribute the lateral wave field and gain saturation in the MMI coupler to produce nonlinear coupling, power-dependent switching was observed. Such a nonlinear device could be modulated either by electrical currents or optical signals. The results of numerical simulations agreed well with the experimental data. Since the dynamics of a semiconductor optical amplifier is controlled by the carrier lifetime, the operation speed is limited by this parameter. This limitation applies to all NOLM's and similar devices based on absorption/gain saturation in semiconductor.

\section{REFERENCES}

[1] J. Cai, K. Feng, X. P. Chen, and A. E. Willner, "Equalization of nonuniform EDFA gain using a fiber-loop mirror," IEEE Photon. Technol. Lett., vol. 9, pp. 916-918, 1997

[2] W. S. Wong, S. Namiki, M. Margalit, H. A. Haus, and P. Ippen, "Selfswitching of optical pulses in dispersion-imbalanced nonlinear loop mirrors," Opt. Lett., vol. 22, pp. 1150-1152, 1997.

[3] D. Wang, E. A. Golovchenko, A. N. Pilipetskii, C. R. Menyuk, and M. F. Arend, "Nonlinear optical loop mirror based on standard communication fiber," J. Lightwave Technol., vol. 15, pp. 642-646, 1997.

[4] J. P. Sokoloff, P. R. Prucnal, I. Glesk, and M. Kane, "A terahertz optical asymmetric demultiplexer (TOAD)," IEEE Photon. Technol. Lett., vol. 5, pp. 787-790, 1993.

[5] M. Eiselt, W. Pieper, and H. G. Weber, "SLALOM: Semiconductor laser amplifier in a loop mirror," J. Lightwave Technol., vol. 13, pp. 2099-2112, 1995.

[6] E. Jahn, N. Agrawal, W. Pieper, H. J. Ehrke, D. Franke, W. Furst, and C. M. Weinert, "Monolithically integrated nonlinear Sagnac interferometer and its application as a $20 \mathrm{Gbit} / \mathrm{s}$ all-optical demultiplexer," Electron. Lett., vol. 32, pp. 782-784, 1996.

[7] C.-H. Tsai, J.-H. Lee, H.-J. Chiang, C. C. Yang, M. C. Shih, B. C. Chen, T. J. Chuang, and Y. Chang, "Semiconductor circular ring lasers fabricated with the cryo-etching technique," IEEE Photon. Technol. Lett., vol. 10, pp. 751-753, 1998.

[8] P. LiKamWa, A. Miller, C. B. Park, J. S. Roberts, and P. N. Robson, "All optical switching of picosecond pulses in GaAs quantum well waveguide coupler," Appl. Phys. Lett., vol. 57, pp. 1846-1849, 1990. 